Entwicklung des Umweltschutzes bei der Dr. Oetker Nahrungsmittel KG

\section{Einbindung der Mitarbeiter}

\author{
Ein sehr wichtiger Erfolgsfaktor für die Umsetzung des betrieblichen Umwelt- \\ schutzes bei Dr. Oetker war die frühzeitige Einbindung der Mitarbeiter. Der \\ Autor, Umweltschutzbeauftrager der Firma, berichtet über Beginn und heutigen \\ Stand des Umweltmanagements in dem Unternehmen.
}

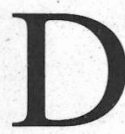

Von Günter Larisch mittel KG ist mit etwa 2.800 Mitarbeitern und acht inländischen Produktionsstandorten eines der großen und erfolgreichen Unternehmen der Ernährungsindustrie. Produziert wird ein breites Sortiment, das von Backzutaten, Puddingpulvern, Müslis über Eiskrem und Honig bis hin zu Tiefkühlprodukten wie Pizza oder Backwaren reicht. Die Geschichte des Umweltschutzes bei Dr. Oetker begann im Jahre 1987, als Herr Oetker von einer Veranstaltung kam, in der Herr Dr. Winter, Gründer der Umweltorganisation B.A.U.M., über Umweltschutz, Ressourcenschonung und Umweltmanagement gesprochen hatte. Das war eigentlich der Auslöser für Herrn Oetker, basierend auf einem ethischen Grundverständnis zur Erhaltung von Natur und Lebewesen, so etwas auch bei uns einzuführen.

In der Startphase haben wir im Einkauf begonnen, weil wir die Meinung vertreten haben, im Einkauf könne man schon sehr früh durch den Einfluß auf Lieferanten, durch Kauf oder durch Nichtkauf bestimmter Produkte, den ersten Schritt zu einer umweltgerechten Produktion tun. Das habe ich drei Jahre neben meinen Aufgaben als Hauptabteilungsleiter „Einkauf“" gemacht und 1987 praktisch bei Null begonnen. Diese ersten drei Jahre waren sehr mühevoll, denn wir konnten kaum auf etwas Bestehendes im Umweltschutz zurückgreifen. Ich mußte mich schon sehr intensiv bemühen, um über den Aufbau eines Umweltmanagements brauchbare Hinweise zu bekommen. So habe ich mir - besonders hat mir damals die IHK Bielefeld wertvolle Hilfe geleistet - bei Firmen, die von der
Gesetzgebung her Beauftragte stellen mußten, die ersten wichtigen Informationen beschafft. Begonnen habe ich meine aktive Umweltarbeit 1987 mit einer IstAnalyse der Umweltsituation in Produktion und Verwaltung. Daraus ergaben sich erste Maßnahmen, die ich mit Hilfe eines Arbeitskreises umzusetzen begann.

\section{- Einbindung der Mitarbeiter von oben nach unten}

Besonders wichtig ist die Einbindung der Mitarbeiter in den betrieblichen Umweltschutz. Natürlich wollen die Mitarbeiter einem Unternehmen angehören, das Umweltschutz praktiziert und vorlebt. Deswegen ist einer meiner wichtigsten Grundsätze: Umweltschutz muß von oben nach unten gehen. Wir müssen gute Beispiele geben. Das ist das eine. Das andere ist natürlich, daß wir die Mitarbeiter durch gezielte Informationen motivieren, Eigenleistungen im Umweltschutz zu erbringen. Wir müssen jedem Mitarbeiter klarmachen, da $\beta$ er eine Eigenverantwortung hat und daß er selber an der positiven Entwicklung des Umweltschutzes im Unternehmen und auch darüber hinaus aktiv mitwirken kann.

Wir haben daher in der zurückliegenden Zeit viel unternommen, um die Mitarbeiter in den aktiven Umweltschutz einzubinden. Zunächst gilt es, ausführlich und umfassend zu informieren. Jeder sollte wissen, was wir tun und was wir tun wollen. Dafür sind die Umweltberichte vorgesehen. Wir geben diese Umweltberichte jedem Mitarbeiter in Verbindung mit einem Umweltquiz, so daß wir ziemlich sicher sein können, daß sie auch gelesen werden. Wir nutzen zusätzlich zur Information unsere Mitarbeiterzeitung „Dr.
Oetker Nachrichten“, in der seit einiger Zeit auch regelmäßig Umweltthemen behandelt werden. Wir informieren seit ungefähr neun Monaten durch unser Rundschreiben „Umwelt-Forum“, über umweltrelevante Entwicklungen in unserem Unternehmen und darüber hinaus. Es werden monatlich bestimmte Umweltthemen, die teilweise auch in den privaten Bereich der Mitarbeiter gehen, behandelt.

\section{Aus- und Weiterbildung}

Jeder Jahrgang unserer Auszubildenden führt ein Umwelt-Projekt durch, das dann der Geschäftsführung präsentiert wird. Bisher wurden alle Empfehlungen im Unternehmen umgesetzt. Das motiviert ungeheuer und prägt für die Zukunft das Umweltbewußtsein der jungen Mitarbeiter. Themen der Umweltprojekte waren zum Beispiel:

- das umweltfreundliche Büro,

- Entwicklung eines Farbleitsystems für Abfälle,

- Energie- und Wassereinsparungspotentiale in Produktion und Verwaltung.

Zur Motivation dient ferner das „Betriebliche Vorschlagswesen“. Die Mitarbeiter können Umweltverbesserungsvorschläge einreichen. Es gibt eine Formel, nach der im Verhältnis zum Nutzen für das Unternehmen Prämien ausgezahlt werden. Verbesserungsvorschläge im Umweltschutz schlagen sich aber nicht immer als finanzielle Einsparung nieder. Deshalb erhalten die Mitarbeiter in jedem Fall für einen Verbesserungsvorschlag einen Sachpreis als Belohnung für ihre Aktivität.

Des weiteren werden interne Schulungen durchgeführt, die wir oft selbst leiten, weil die Inhalte zum großen Teil unternehmensbezogen sind. Wir haben in den letzten Jahren zum Beispiel eine Schulung „Umweltbewußtseinsbildung“ als Work-shop angeboten sowie Schulungen im Abfall- und Gefahrstoffbereich durchgeführt. Wenn uns für Schulungen das notwendige Know-how fehlt, wird die Weiterbildung von externen Beratern durch- geführt, so zum Beispiel die Ausbildung zum internen Umweltauditor. 


\section{- Beteiligung an internen Audits}

Dann gibt es noch die aktive Beteiligung der Mitarbeiter an unseren internen Umweltaudits, die wir seit einigen Jahren mit eigenen Leuten durchführen. Unsere internen Umweltaudits sind so aufgebaut, daß sie der EG-Öko-Audit-Verordnung entsprechen. Durch einen innerhalb des Audits eingebundenen Workshop und ausführliche Interviews versuchen wir auch die Probleme der Mitarbeiter in Erfahrung zu bringen, um sie ggf. mit in den Maßnahmenkatolog aufzunehmen. Wenn wir die vorläufigen Ergebnisse am Schluß des Umwelt-Audits vorstellen, beginnen wir zur Motivation mit den festgestellten Stärken im Umweltschutz. Wir stellen dann anschließend natürlich auch die ermittelten Mängel vor, denn die gilt es ja zu verbessern. Wir kontrollieren nicht, sondern wir ermitteln den IstZustand. Daraus werden Maßnahmen entwickelt, die letztendlich zur Verbesserung der Umweltsituation führen sollen. Die Maßnahmen werden in einem Maßnahmenkatalog festgehalten und nach ca. 6 Monaten auf ihre Umsetzung überprüft. Alle unsere Standorte wurden bereits mindestens einmal intern auditiert. Im Mai '97 wurde unser Werk in Wittenburg nach EMAS validiert. Im Herbst '97 folgt unser Werk in Bielefeld. Danach wird der Geschäftsführung eine ausführliche Stellungnahme über die bisherigen Erkenntnisse vorgelegt und die weitere Vorgehensweise in Sachen Öko-Audit abgestimmt.

\section{Umweltmitarbeitererklärung}

Neue Mitarbeiter, die bei Dr. Oetker anfangen, müssen sich auf das Unternehmensziel „Umweltschutz" verpflichten. Vor ca. 3 Jahren haben wir eine Umwelt-

\section{Der Autor}

Günter Larisch ist Umweltbeauffragter bei der Dr. Oetker Nahrungsmitiel KG

Kontakt: Dr. Oetker Nahrungsmittel KG, Luttersir. 14, 33617 Bielefeld, Tel. 0521/1552561 Fax $0521 / 152900$ mitarbeitererklärung eingeführt, die jeder neue Mitarbeiter, auch ein Praktikant oder Student, der seine Diplomarbeit schreibt, aber auch ein Geschäftsführer, mit seinem Einstellungsvertrag zu unterschreiben hat. Diese Maßgabe gilt für alle neuen Mitarbeiter und hat sich inzwischen gut eingespielt. Wir überlegen zur Zeit, diese Umwelterklärung von allen Mitarbeitern unterschreiben zu lassen.

Sehr wichtig für den Erfolg im Umweltschutz ist die Miteinbeziehung des Managements in die aktive Umweltarbeit. Deshalb war es auch immer mein besonderes Anliegen, bei den Führungskräften Verständnis für meine Aufgaben als Umweltbeauftragter zu erlangen. Da ich schon sehr lange im Unternehmen und als ausdauernder aber auch energischer Verfechter meiner Ziele bekannt bin, komme ich gut voran und meine Arbeit wird akzeptiert und inzwischen auch für notwendig gehalten. Es ist von großem Vorteil, daß Herr Oetker und die gesamte Geschäftsführung den Umweltschutz im Unternehmen sehr hoch eingestuft haben.

\section{Veto-Recht für den Umweltschutz}

Um Unternehmensentwicklungen und geplante Investitionen im Sinne des Umweltschutzes mitbeeinflussen zu können, besitze ich als Umweltbeauftragter ein Veto-Recht und kann somit umweltrelevante Vorgänge stoppen, um sie einer weiteren genauen Prüfung zu unterziehen. Wenn ich also bestimmte Informationen erhalte oder etwas höre oder sehe, das nicht mit unserer Umweltpolitik übereinstimmt, werde ich aktiv. Dieses Vetorecht wurde bereits bei Einkaufsvorgängen, unter anderem beim Bezug von Tiefkühlgeräten und beim Einsatz von Packstoffen, erfolgreich angewandt. Ein derartiges Veto kann nur durch einen Beschluß der Geschäftsleitung aufgehoben werden. Wir sind eine der wenigen Firmen, in denen der Umweltbeauftragte ein Veto-Recht besitzt.

$\mathrm{Zu}$ einem aktiven Umweltschutz gehört auch die Einbeziehung der Lieferanten. Deshalb haben wir mit dem Einkauf sowie mit Forschung und Entwicklung im letzten Jahr vereinbart, mittels Umweltchecklisten unsere wichtigen Lieferanten unter Umweltgesichtspunkten zu überprüfen. Wir haben ein System entwickelt, um die Umweltbeurteilungen in die Gesamtbeurteilung der Lieferanten miteinfließen zu lassen. Ein Lieferant wurde bisher nach Qualität, Pünktlichkeit und Preiswürdigkeit bewertet. Nun ist als weiteres Merkmal - wie bereits erwähnt - die Leistung im Umweltschutz hinzugekommen.

Natürlich kann Umweltschutz auch mit dazu beitragen, wirtschaftlichen Nutzen und Wettbewerbsvorteile für ein Unternehmen zu erzielen. Besonders wichtig ist jedoch, daß es im Umweltschutz Vorreiter und Nachahmer gibt, denn nur in der Summe vieler Aktivitäten hat der Umweltschutz die Chance, wirklich erfolgreich zu sein und Entscheidendes zu bewegen.

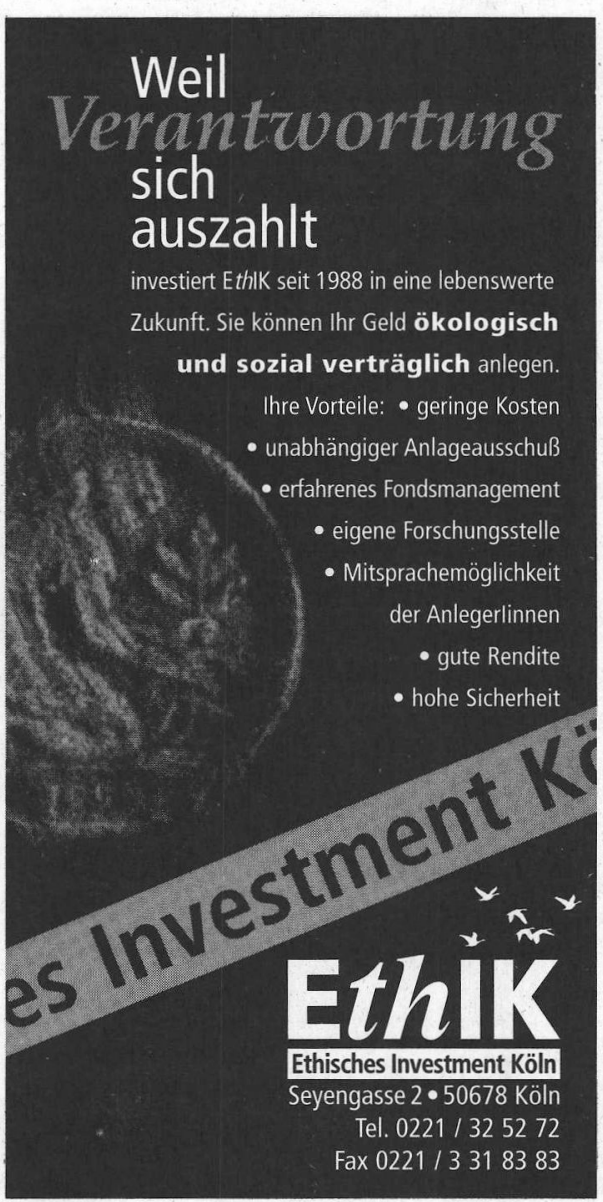


(c) 20I0 Authors; licensee IÖW and oekom verlag. This is an article distributed under the terms of the Creative Commons Attribution Non-Commercial No Derivates License (http://creativecommons.org/licenses/by-nc-nd/3.o/), which permits unrestricted use, distribution, and reproduction in any medium, provided the original work is properly cited. 